\title{
IJMOR
}

International Journal of Medical and Clinical Research

ISSN: 0976-5530 \& E-ISSN: 0976-5549, Volume 2, Issue 2, 2011, pp-63-66

Available online at http://www.bioinfo.in/contents.php?id=39

\section{LAPAROSCOPY IN 50 INFERTILE COUPLES: PROSPECTIVE STUDY}

\author{
BORICHA Y.G., SHARMA R.K.*, BORICHA B.G., SABRINA MHAPANKAR, ARCHANA CHATERJEE, \\ NARSHETTY J. \\ Department of Obstetrics and Gynecology, MGM Medical College, Kamothe, Navi Mumbai \\ *Corresponding author. E-mail: drrksharmaz@gmail.com
}

Received: October 06, 2011; Accepted: November 02, 2011

\begin{abstract}
Use of diagnostic and therapeutic laparoscopy in infertility has been a focus of attention in recent years and demonstrated to be very effective method in evaluating these cases.. In our study, we were able to carry out therapeutic intervention in 22 cases $(61.1 \%)$. It is observed that diagnostic laparoscopy is indicated in all cases of bilateral tubal anomalies and has identified important pelvic pathology in significant number of selected infertile patients even after normal findings. It is concluded that laparoscopy is useful in diagnosing cases with endometriosis and tubal factor infertility and exclusion of bilateral anatomical tubal pathology by diagnostic laparoscopy could avoid IVF treatment in these cases. The importance \& scope of newer procedure like fertiloscopy along with laparoscopic evaluation is also discussed.
\end{abstract}

Keywords- female infertility, IVF, laproscopic techniques, pelvic pathology

\section{Introduction}

Approximately $85-90 \%$ of healthy young couples conceive within one year. Infertility therefore, affects approximately $10-15 \%$ of couples and is an important part of clinical practice for many clinicians. The female factors contribute most (i.e. $40-55 \%$ ) in the etiologies of infertility followed by male factors $(30-40 \%)$, both partners (10\%) and unexplained (10\%)[1]. Advances in endoscopic surgery have revolutionized the approaches of obstetricians for diagnosis and management of patients with infertility. Major causes of infertility include ovarian dysfunction, tubal disease, endometriosis, uterine or cervical factors and male factors. Contrary to popular perception, the overall incidence of infertility has remained relatively unchanged over the past three decades. However, the evaluation and treatment of infertility have changed dramatically during that time. Corson SL, Cheng A, Gutmann JN (2000)[2] studied role of laparoscopy in 100 infertile patients with normal findings and reported that Out of these, 68 patients had pathologies of reproductive significance $(68 \%)$. In a retrospective study, Boudhraa $K$, Jellouli MA et al., (2009)[3] evaluated 200 cases of hysterolaparoscopy in female infertility patients. He reported that diagnostic laparoscopy after several failed cycles of ovulation induction enabled the detection of a significant proportion of pelvic pathology amenable to treatment. A Cochrane review has shown that laparoscopic ovarian diathermy in clomiphene-resistant polycystic ovarian syndrome is at least as effective as gonadotrophin treatment, and results in a lower multiple pregnancy rate. Kanal $P$, Sharma S (2006)[4] concluded that Laparoscopy combined with Hysterosalpingography (HSG) is more effective method to reveal tubal blocks. However they suggested that hysterosalpingo-contrast-sonography (HyCoSy) was a cost effective screening test as compared to diagnostic laparoscopy with HSG in the assessment of tubal patency for the investigations of infertility patients. Today, Laparoscopy still represents the second option after several failures of in vivo attempts and before moving to conceive in vitro. Bulletti C, Panzini I et al., (2008) [5] studied the diagnostic power of hysterosalpingography and hysterosalpingosonography compared with that of Laparoscopy and the efficacy of ART when each single test is used as an inclusion criterion and concluded that, Pelvic factors other than tubal occlusions are neither diagnosed nor treated in a timely manner by indirect tubal patency tests. The conventional use of hysterosalpingography and or hysterosalpingosonography may increase the time required to find an adequate treatment by which to achieve a successful pregnancy. Sakar MN, Gul T, Atay $A E$, Celik Y, (2008)[6] compared the tuboperitoneal factors of infertile women by hysterosalpingography (HSG) and laparoscopy. In their cohort study, 82 infertile cases were evaluated retrospectively by laparoscopy, 3 months subsequent to HSG. It is reported that the sensitivity of HSG was $63 \%$, specificity was $89.3 \%$, and the positive predictive value was $92 \%$, with a $55 \%$ predictive value, and the accuracy ratio was $72 \%$. Further it was concluded that laparoscopy was a superior method for the research of tubal and pelvic pathologies in the evaluation of infertility. However, HSG is a more 
economical and elementary method suitable for evaluation of endometrial and tubal pathologies, and laparoscopy is an appropriate method for examining the external part of tube, fimbriae, the relation of tubes and ovary, endometriosis, adhesions, tuberculosis, and other pathologies. Therefore, these 2 methods are not alternative, but complementary. Sharon E M, Henry C. L et al., (2009) [7] conducted a cost-effectiveness analysis using a computer-generated decision analysis tree and observed that laparoscopy with expectant management (LSC/EM) was preferred (ICER $=\$ 128,400$ per live-birth in U.S. dollars) as compared to standard infertility treatment algorithm (SITA) and laparoscopy with infertility therapy (LSC/IT) and concluded that laparoscopy is cost effective in the initial management of young women with infertility, particularly when infertility treatment dropout rates exceed $9 \%$ per cycle. Thus advances in laparoscopic-surgical technology as well as alternative diagnostic methods for diagnosis and management of female infertility is subject of debate and desire a comprehensive evaluation prior to use for the diagnosis of infertility and revolutionized the treatment. Present study has been undertaken to ascertain the diagnostic and therapeutic role of laparoscopic technique in patients of female infertility attending the tertiary care hospital at Navi Mumbai.

\section{Material and methods}

The present study carried out from May 2008 to September 2009 and evaluated 50 couples having infertility, selected by complete numeration method and by using a structured questionnaire and comprehensive consent form to avoid personal bias. Written consent of the patient \& her relative was taken.

Fifty cases of primary and secondary infertility were included in the study. The patients having heart disease, chromosomal disorder, schizophrenia, known cases of uterine perforations in past, documented $\mathrm{H} / \mathrm{O}$ Genital tuberculosis, uterine anomalies as diagnosed on HSG were excluded from the study. Patients with all contraindications as related to procedure of laparoscopy like, generalized peritonitis, bowel obstruction, hernia, tuberculus peritonitis with adhesions, large pelvic mass were also excluded from the study.

\section{Method}

Laparoscopy was done under controlled general anesthesia. The patient was asked to pass urine before the procedure. The patient was placed in lithotomy position. A crescentic incision was made in the infraumbilical fold. Pneumoperitoneum was created with veress needle. After confirming the correct placement of the needle, $\mathrm{CO}_{2}$ gas is insufflated into the peritoneal cavity. The gas is insufflated at the rate of $1 \mathrm{~L} / \mathrm{min}$, a total of 2-3 $L$ of gas being required. The intra-peritoneal pressure during insufflation is $10-15 \mathrm{~mm}$ of $\mathrm{Hg}$. It is $5 \mathrm{~mm}$ of $\mathrm{Hg}$ with good muscle relaxation. Creation of a satisfactory pneumoperitoneum is confirmed by noting uniform distension of the abdomen \& obliteration of liver dullness.
The trocar \& cannula are introduced through crescentic incision in the infra umbilical fold, elevating the abdominal wall, the tip being directed towards the sacral hollow. The passage is zigzag through the rectus sheath to reduce the risk of herniation of the intra-abdominal contents through the tract after laparoscopy. Entry into the peritoneal cavity is signaled by escape of gas. The trocar is removed \& the laparoscope is inserted. The fiber optic light cable is connected to the laparoscope \& the light source. The camera is also connected. The intra peritoneal structure is then inspected thoroughly, evaluating the peritoneum, uterus, both ovaries, both fallopian tubes etc. to note for any abnormalities. A $5 \mathrm{~mm}$ trocar \& cannula for $2^{\text {nd }}$ puncture instrumentation is inserted through a suprapubic / suprailiac transverse incision. Through this a manipulating blunt probe can be passed for manipulation as well as measuring the size of intraperitoneal structures. Other $2^{\text {nd }}$ puncture instruments that can be used are silastic band applicator, scissors, knife, aspiration needle, electrode for electrocautrization, biopsy forceps \& forceps with blunt jaw for holding structures like the round ligament or utero-ovarian ligament. Tubal patency is tested by chromopertubation with methylene blue dye, diluted and injected in the uterine cavity through HSG cannula, transcervically.

After the procedure, irrigation and suction is done, secondary ports are removed under vision to watch for active bleeding from the puncture site. After confirming hemostasis the laparoscope is removed, the trocar is inserted in the cannula \& the gas is removed from the peritoneal cavity after making the patient supine. Then the trocar \& cannula are removed \& the incision is closed with the single stitch of linen, silk or metal clips to be removed after 7 days.

Postoperative management of the patients included analgesics for epigastric \& shoulder pain due to residual gas in peritoneal cavity, and antibiotic cover. Patients were discharged after 24 hours of observation, if stable.

All the patients were asked to follow up in OPD after 7 days for removal of skin sutures.

All the data were computerized for analysis using Microsoft excel system.

\section{Observations}

We studied 50 patients of female infertility comprising of $35(70 \%)$ cases of primary infertility and $15(30 \%)$ cases of secondary infertility

The duration of infertility ranged from 1.5 to 12 years. Maximum number of cases had duration of infertility between 4 to 7 years, in both primary infertility group- 19 cases $(54.28 \%)$ and secondary infertility group- 7 cases $(46.66 \%)$. The mean duration of infertility was found to be 4.2 years in cases of primary infertility and 4.8 years in cases of secondary infertility.

In cases of primary infertility most of the patients were from the age group of 21 to 25 years, 15 cases (42.85\%) and 26 to 30 years, 6 cases (40\%) in cases of secondary infertility. The mean age at presentation was 26.5 years and 30 years for primary and secondary infertility respectively. 
Our study reveals that in cases of primary infertility, the commonest finding was ovarian pathology affecting 13 cases (37.14\%). Tubal, ovarian and endometriosis were seen in equal number of cases in patients with secondary infertility 3 cases (20\%), with normal findings being the commonest 5 cases $(33.33 \%)$.

However, considering all the 50 cases, the most common finding on laparoscopy was found to be ovarian factor, affecting 16 cases (32\%). Out of the 16 cases $(32 \%)$ with ovarian pathology, polycystic ovarian disease was found to be the commonest 8 cases (50\%), followed by simple ovarian cyst and chocolate cyst.

Other findings were endometriosis 11 cases $(22 \%)$, tubal pathology 10 cases $(20 \%)$, uterine pathology 9 cases $(18 \%)$ and peritoneal factor in 6 cases $(12 \%)$. Endometriosis was seen in $11(22 \%)$ cases, out of which, according to American Fertility Society Classification, 7 cases $(63.63 \%)$ had mild and 4 cases $(36.36 \%)$ had severe endometriosis. In 3 cases ovaries were involved in the form of chocolate cyst, in rest of the cases peritoneum, uterosacral ligaments, fallopian tube, adnexa were involved. Dense adhesions with obliteration of pouch of Douglas were seen in only one case.

Out of the 10 cases (20\%) with tubal pathology, 7 cases (70\%) had unilateral block, and in 2 cases (20\%) both the tubes were blocked. Hydrosalpinx was most commonly found. Other findings were cornual block, terminal block and fimbrial agglutination. In 1 case, fallopian tube was absent on one side due to previous salpingectomy for ectopic pregnancy. Previous pelvic inflammatory disease was not an uncommon factor responsible of tubal blocks. In one case genital Koch's was diagnosed as a cause for tubal block.

In cases with uterine findings (18\%), anomalies were seen in 6 cases $(66.66 \%)$ and fibroids were seen in 4 cases $(44.44 \%)$.

Laparoscopy showed normal findings in 14 cases (28\%) and did not demonstrated evidence of any pathology. 2 of these cases had male factor infertility and 1 case was diagnosed of other pathology. A total of 11 cases $(22 \%)$ had unexplained infertility ith no obvious cause seen in both partners.

Peritoneal factors were observed in 6 cases $(12 \%)$, with adhesions in pouch of Douglas being the frequently encountered finding. Endometriosis involving the peritoneum leading to adhesions, along with previous pelvic inflammatory disease was the common etiological factors.

The patients with pathological finding were subjected to therapeutic interventions. Out of the 36 cases $(72 \%)$ with some pathological finding noted during laparoscopy, therapeutic intervention was done in 22 cases $(61.11 \%)$. Which included; ovarian drilling 9 cases $(40.90 \%)$, cyst puncture 5 cases $(22.72 \%)$, excision and cauterization of chocolate cyst 3 cases (13.63\%), adhesiolysis 3 cases $(13.63 \%)$, hydropertubation 3 cases $(13.63 \%)$, ablation of endometriotic spots etc. In one case, tubal cannulation was attempted for bilateral blocked tubes, but failed. It was noted that there were successful pregnancies in 1 case after ovarian drilling and 1 case after adhesiolysis.
The major complications encountered were abdominal wall injury in 1 case (2\%), surgical emphysema in 1 case (2\%), injury to mesosalpingeal vessel 1 case $(2 \%)$. All injuries were of mild grade and did not require any active intervention. In 1 case (2\%), patient developed breathing difficulty immediately after surgery and required reintubation. Anesthetic complication was suspected in this case. Spontaneous respiration was regained after half an hour and period of stay in hospital was increased by 3 days. Long-term complications like incisional hernia could not be assessed adequately in all cases due to cases lost to follow-up, although it was not seen in any cases till the end of our study period.

Out of the 50 study cases, 15 cases (30\%) were lost to follow-up. From the rest of the 35 cases $(70 \%)$ who followed up regularly, 3 patients were referred to higher centre for In Vitro Fertilization and Embryo Transfer in view of bilateral tubal block in 2 cases, and unilateral tubal block with failure to conceive after ovulation induction and intra-uterine insemination, in 1 case. 1 patient was started on AKT for 6 months after diagnosing genital tuberculosis. 7 patients $(20 \%)$ out of the 35 , who followed up regularly and were treated, conceived. Therapeutic intervention were done in 3 of these cases, rest of them had infertility of unknown etiology and conceived after ovulation induction with intra-uterine insemination for an average of 2 to 3 cycles.

\section{Discussion}

In our study, an effort was made to find out the role of laparoscopy in infertile females and to detect its diagnostic and therapeutic efficacy. A total of 50 infertile women were studied over a span of one and a half year. The mean age at presentation in our study was 26.5 years (range of $21-25$ years) in primary infertility and 30 years (range of 26- 30 years) in secondary infertility group i.e. just beyond the maximum fertile period of life. This is consistent with the observations by Waseem $T$ et a/8. Buyalos, Daneshmand and Brzechiffa (1997)9 investigated the fertility of women of different ages and observed that a woman reaches her maximum fertility potential at the age of 24 years. Fertility potential begins to decline from the age of 30 years [8]. The mean duration of infertility was 4.2 years for the group with primary infertility and 4.8 years for secondary infertility group (range of 4- 7 years for both groups). The study by Waseem $T$ et al., found the same to be 6.1 years and 4.3 years respectively. It is also reported that most common etiological factor too was ovarian[8]. The incidence of endometriosis has almost remained unchanged. There has been a decline in tubal pathology possibly due to decreased incidence of infection and timely treatment with antibiotics. We also observed that ovarian factors were found to be the most common finding $(32 \%)$, with PCOD affecting $50 \%$ of these patients. Other common pathologies found were endometriosis $(22 \%)$, tubal pathologies $(20 \%)$, uterine pathologies $(18 \%)$ and peritoneal factors $(12 \%)$. The normal laparoscopic findings were recorded in $28 \%$ of cases. Present study correlated with observation of the other studies 
suggesting that Laparoscopy was useful in achieving a correct diagnosis, especially in cases of endometriosis and peritoneal factor infertility, which were poorly diagnosed prior to the procedure. Our study proved laparoscopy as useful tool in carrying out therapeutic intervention in 22 cases (61.1\%). Ovarian drilling was carried out in 9 cases that had not responded to Clomiphene citrate induction of ovulation prior to the surgery. Many studies [10,11, 12] evaluated the effect of ovarian electro cauterization and ovarian response to gonadotrophin stimulation and pregnancy rate in clomiphene citrate resistant PCOS and recorded reduced basal serum LH concentration and normal cyclity in $41 \%$ patients after ovarian drilling. Comparison of gonadotrophin stimulated cycle before and after electro cauterization revealed higher rates of ovulation and pregnancy after drilling as well as significant reduction of gonadotrophin ampoules. They concluded that there was an increase in ovarian sensitivity to gonadotropins after laparoscopic ovarian drilling. Garry et al in their study concluded that meaningful improvements in clinical symptoms of quality of life can be obtained by laparoscopic excision of endometriosis and endometriomas with acceptable levels of operative morbidity[13]. In our study, out of the 7 cases $(20 \%)$ that conceived after treatment, $3(42.85 \%)$ had undergone some therapeutic intervention during laparoscopy. Improvement in the diagnostic accuracy and possibility of therapeutic intervention with laparoscopy improved the overall scope of planning the patient's further management. In 20 cases $(40 \%)$, the plan of management changed after laparoscopy. 7 cases (20\%) out of the 35 who followed up and were treated, conceived. This is consistent with the study by Pelinck $\mathrm{MJ}$ et al, who found the pregnancy rate to be $20-25 \%$ in women treated for infertility either by conservative or artificial reproductive techniques, in their study[14].

\section{Conclusion}

Infertility affects about $10-15 \%$ of the population. Laparoscopy is a very effective method in evaluating these cases. In about half of the cases, the diagnosis was corrected because of laparoscopy. Diagnostic laparoscopy is indicated in all cases of bilateral tubal anomalies. Exclusion of bilateral anatomical tubal pathology by diagnostic laparoscopy could avoid IVF treatment in these cases. Also, it may be considered in appropriately selected infertile patients even after normal findings, as important pelvic pathology may be identified in a significant number of patients. It is most useful in diagnosing cases with endometriosis and tubal factor infertility. In our study, we were able to carry out therapeutic intervention in 22 cases (61.1\%). Advances in endoscopic surgery have also revolutionized our approaches to patients with infertility. Ideally, all reproductive operations should be done via laparoscopy. The variety of conditions that can be corrected by laparoscopic surgery emphasises the importance of acquiring laparoscopic-surgical skills in the practice of reproductive medicine. It's only then, that the patients can be offered the most appropriate treatment. It is a known fact that, endoscopic surgery for infertility patients, when performed, by an experienced endoscopist, is efficacious and can produce as good or better results than traditional procedures.

Laparoscopic evaluation in infertility now also includes newer procedures like fertiloscopy. Although, diagnostic laparoscopy is simple, safe, available and diagnostically accurate, it is an invasive and traumatic procedure. Although the recorded mortality in most of the centres across the world remains to be zero, it does carry the potential risk of mortality and morbidity. It should not be the first method to diagnose the cause of infertility. It should be reserved for those situations, where other methods have failed to give substantial information of the cause of infertility.

\section{References}

[1] Speroff L., Marc A. F. (2005) Female infertility: Clinical Gynecologic Endocrinology and Infertility, $7^{\text {th }}$ Ed, Jaypee Brothers Medical Publishers, India; 1013-1068.

[2] Corson S.L., Cheng A., Gutmann J.N. (2000) J Am Assoc Gynecol Laparosc; 7(3): 317-324.

[3] Boudhraa K., Jellouli M.A., Kassaoui O., Ben Aissia N., Ouerhani R., Triki A., Gara M.F. (2009) Tunis Med; 87(1): 55-60.

[4] Kanal P., Sharma S. (2006) Internet Journal of Medical Update; 1(2): 7-9.

[5] Bulletti C., Panzini I., Borini A., Coccia E., Setti P.L., Palagiano A. (2008) Ann N Y Acad Sci; 1127: 73-82

[6] Sakar M.N., Gul T., Atay A.E., Celik Y.(2008) Saudi Med J; 29(9): 1315-1318.

[7] Sharon E. M., Henry C. L., Ruth B. L., Lynn M. W., Amin A. M., Alan M. G. (2009) Fertility and Sterility; 92(2): 471-480.

[8] Waseem T., Mohammad I., Maimoona H., Mohammad S. (2007) Professional Med J; 14(4): 562-566.

[9] Buyalos R.P., Daneshmand S., Brzechiffa B. (1997) Fertility and sterility; 68: 272-277.

[10] Farhi J. S. et al. (1995) Fertil .Steril; 64: 930935.

[11] Farquhar C., Lilford R.J., Marjoribanks J., Vandekerckhove P. (2005) Cochrane Database Syst Rev; 3:CD001122.

[12] Hassan Al-Ojaimi E.(2003) Bahrain Medical Bulletin; 25(2): 58-63.

[13] Garry R., Clayton R., Hawe J. (2000) Br J Obstet Gynaecol; 107(1): 44-45.

[14] Pelinck M.J., Vogel N.E.A., Hoek A., Simons A.H.M., Arts E.G.J.M., Mochtar M.H., Beemsterboer S., Hondelink M.N. and Heineman M.J. (2006) Human Reproduction; 21(9): 2375-2383. 